\title{
Practical Aspects of the Dependence of Polarity on Temperature
}

\author{
K. Grob / G. Grob \\ GC-Laboratory, ETH Zürich; EAWAG; CH-8600 Dübendorf, Switzerland
}

\section{Key Words}

Polarity

Stationary phases

Temperature dependence

GC

\section{Summary}

It is not commonly appreciated that retention indices are temperature dependent. It is even less common to express this fact in more practical terms by saying that polarity is temperature dependent. Although the meaning of both statements is identical, we believe the second to be particularly relevant, since the majority of practical gas chromatographers tends to handle polarity as an invariable characteristic of a stationary phase.

The variability of polarity with temperature is the major source of inadequate reproducibility of exact fingerprinting, this particularly when $\mathrm{gc} / \mathrm{ms}$ traces have to be compared to those obtained by pure gc. On the other hand, the temperature dependence provides a practical means to optimize the polarity of a given column for a given analysis. Film thickness is an essential parameter in this context because of its influence on column temperature and, therefore, on column polarity.

\section{Introduction}

Our somewhat embarassing purpose is to treat a subject which is, on the one hand scientifically known or even trivial but, on the other hand, unappreciated by a majority of practical chromatographers. The practical usefulness causes us to treat the subject differently as compared to the usual literature.

Polarity has always been one of the most fundamental concepts of gas chromatography. As we have experienced on a large number of introductory courses, virtually all chromatographers think of polarity as a phenomenon of very complex origin, but as an absolutely typical property of a stationary phase and, therefore, a constant characteristic of that phase. They are shocked to hear that polarity is a function of temperature, which means that it is a variable attribute of a given phase. With surprise they learn that the scientific background of the fact has been fully available for years, and that their unawareness is probably due to a way of presentation which is correct from a scientific point of view but unfortunate from a practical one.

\section{Retention Indices and Temperature}

We are far from attempting a complete or, indeed, representative presentation of the extremely rich bibliographic background of the subject. By citing selected examples we wish to show that the subject has always been treated in a relatively heterogeneous way which has been hardly suitable for creating straightforward understanding in nonspecialized readers.

The creator of the retention index system, Kovats [1], discusses carefully the influence of temperature on indices and shows ways to account for such influences. Rohrschneider [2] gives no indication concerning the role of temperature. Schomburg [3] mentions a "small temperature dependence, depending upon the type of substance".

In the seventies the literature continues to be rather inhomogeneous. Two papers dealing with the determination of polarity, Lowry et al. [4], and Zielinsky and Martire [5] do not include temperature in their discussion, not even in the discussion of mechanisms of molecular interactions [5]. Rang et al. [6] consider the role of temperature in the determination of retention indices of alkynes. Gross and Weigel [7] utilize the influence of temperature for the specific retention of benzene. A detailed presentation of the role of temperature is given by Sojak and Rijks [8], even discussing differences in the influence of temperature due to ageing of the stationary phase. They also use substance-specific temperature dependencies for the characterization of solutes (see [8] for the previous literature).

In more recent years presentation still remains varying. Schomburg [9] gives a detailed treatment including temperature coefficients for various functional groups on various columns. Lee et al. [10] minimizes the temperature influence by using an index system based on substances closely related to the solutes to be characterized. Jennings [11] not only discusses the temperature dependence of retention indices but also shows temperature to affect the elution of mixtures containing a variety of functional groups. In contrast, Schulte [12] omits temperature in his discussion of the polarity of stationary phases. Knoeppel et al. [13] in their very careful study of retention index determination under programmed temperature conditions do not seem to consider variations of elution temperatures. Korhonen $[14,15]$, despite the drastic temperature program rates he applies, overlooks the correspondingly large variations of elution temperatures and their effects on his columns. 


\section{Retention Indices and Polarity}

The close relationship between a retention index system and polarity has become commonplace, since polarity is best described by the retention indices of a number of suitably selected solutes.

The role of temperature is far less commonly known. In this respect, three practices can be roughly distinguished. The first and most frequent omits the role of temperature. The second practice considers temperature but only as a potential source of errors in the determination of retention indices. The third, and least frequent, practice envisages the full practical role of temperature including its influence on elution order, particularly with substances of different functionality.

Since polarity has always been defined in termes of elution order, a modified elution order produced by the same column at a different column temperature must be attributed to a modified polarity of the same stationary phase. Surprisingly, we have not been able so far to find this simple conclusion in the literature, even not in papers of the third group above in which its full background is present. The particularity of the conclusion is only a matter of formulation. It may, therefore, be interpreted as a play on words. It seems, however, that using the term polarity in this context is of essential practical relevance.

The triviality of the conclusion, combined with an extreme wealth of literature, cause us to think that it may have been expressed previously. What we observe, however, is that it is virtually unused in practical gas chromatography.

\section{The Basic Phenomenon}

To eliminate any misunderstanding, the role of temperature may be demonstrated in a similar way as in references [9] and [11]. Figures $1-3$ present examples in which both the stationary phase and the solutes have been selected rather arbitrarily. On each column the same sample is run at two column temperatures, whereby the slow elution at low temperature is partly balanced by an increased carrier flow.

Fig. 2

As Fig. 1, but OV-1701. Column $14 \mathrm{~m} \times 0.27 \mathrm{~mm}$, persilylated. Substances 1 . bromo nonane, Naphthalene, 1-decanol, dodecanoic acid methylester, $n$-alkanes $\mathrm{C}_{13}-\mathrm{C}_{15}$.

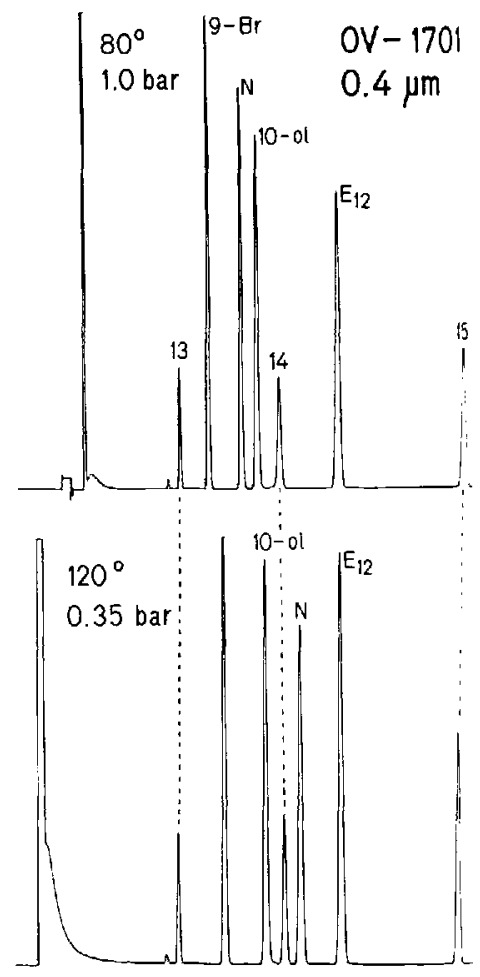

Fig. 3

As Fig. 1 and 2. but OV-17. Column $12 \mathrm{~m} \mathrm{X}$ $0.32 \mathrm{~mm}$, persilylated. For substances see Fig. 2.




Table I. Change of relative retention due to increased column temperature, summary of results of Fig. 1-3.

\begin{tabular}{|lccc|}
\hline Test substance: & \multicolumn{3}{c|}{ Stationary phase: } \\
& PEG & OV 17 & OV.1701 \\
\hline 1-bromoalkane & +4.06 & +2.90 & +3.76 \\
primary alcohol & -1.02 & -0.62 & +5.01 \\
fatty acid methyl & +1.88 & 0.0 & +0.25 \\
ester & +14.7 & +6.55 & +8.57 \\
Imethyl) naphthalene & (values in index units per degree) & \\
\hline
\end{tabular}

Evidently, optimum chromatographic conditions are not attempted. Table I summarizes the results in terms of index units per degree.

When considering polarity as a combined effect of a variety of molecular interactions, the basic influence of temperature is not surprising, since different interactions will hardly show identical dependence on temperature. It would be hard, however, to correlate the results in Table I with the structure of both stationary phases and solutes. The complexity of the situation may be illustrated by an apparently simple example. Aromatic substances are among the solutes exhibiting the strongest response to a change of column temperature. This has probably to be attributed to interactions which are typical for phenyl or polycyclic aromatic groups whereby the aromatic function is not necessarily present in both, solute and stationary phase (see the strong effect on Carbowax). This causes us to expect a correspondingly strong effect for an alcohoi or ester on OV-17, that is, for a combination in which the aromatic function is moved from the solute to the stationary phase. The facts are in complete disagreement with this simple reasoning.

Whereas the examples of Fig. 13 were produced isothermally, analogous effects are obtained with temperature programming. The constant temperature is then replaced by the temperature range within which a component of a mixture migrates over the column. Since there is no simple way of determining this range, the elution temperature of the respective component may be checked. All components of a mixture experience a warmer column (an increased elution temperature) when the temperature program rate is increased, or the carrier flow rate is reduced or when both changes occur simultaneously.

For practical purposes, the situation may be summarized as follows. Changing column temperature, or elution temperature of a given solute means changing the behaviour (the polarity) of the column. For the majority of solute/ stationary phase combinations, increased temperature means increased polarity. Details have to be studied experimentally; rules based on molecular structure should be considered with great caution.

\section{Practical Effects and Applications}

\subsection{Reproducing Fingerprints}

Chromatographers analyzing very complex samples on extremely efficient columns (now commonly available) are familiar with the problem of exactly reproducing the detail- ed elution patterns of a chromatogram. Here a partly resolved peak has disappeared, there a new shoulder has emerged. In earlier years, with less durable columns, this was often due to a permanent polarity change of the column [8]. Now it is normally caused by imprecisely reproduced conditions resulting in a temporary polarity change due to changed column (or elution) temperature. Reproducing the carrier flow rate based on a manometer reading is particularly critical. Setting a constant elution (break through) time of methane, for example, at a given column temperature is more reliable.

\subsection{Comparing GC with GC/MS-Traces}

It is an old problem that chromatograms recorded by FID may greatly differ from what the total ion monitor produces for the same sample and from the same column. Quite often the deviation is tolerated reluctantly as an inevitable consequence of basically changed conditions such as pressure at column exit (atmospheric/vacuum) or nature of the carrier gas $\left(\mathrm{H}_{2} / \mathrm{He}\right)$ or different equipment. Where the change is in fact due to changed conditions, it is fully avoidable.

The working rule for the reproduction of fingerprints is simple. Before switching from gc to $\mathrm{gc} / \mathrm{ms}$, read elution temperatures for a number of major or typical components. On gc/ms vary the carrier flow rate (if necessary also the temperature program rate) up to the level that the elution temperatures are reproduced. Constant elution temperatures indicate constant column polarity resulting in constant elution patterns. Surprisingly, even a drastic pressure change at the column outlet has no effect on its own. A very slight difference may remain when the carrier gas has to be changed [8].

\subsection{Resolving Superimposed Substances}

In discussions among chromatographers on relative merits of various columns, an ever-occurring statement is, "it is a wonderful column but it coelutes X with $\mathrm{Y}$ ". Provided X and $\mathrm{Y}$ are not closely related, or even isomers, we recommend that a different column (elution) temperature be tried. In the majority of cases, $\mathrm{X}$ and $\mathrm{Y}$ are then resolved, without using a different column.

As an example, the naphthalene content in a petrol fraction had to be monitored. An ordinary apolar column eluted naphthalene (Figure 4, chromatogram A, arrow; enlarged presentation on the right) as a shoulder on a neighbouring aliphatic substance. The situation seemed fortunate, since there was almost free space on the chromatogram in the sense of increasing retention. Thus, a slight increase of column polarity was expected to move the naphthalene peak into that open area. Reducing the carrier pressure from 0.5 to 0.4 bar, and increasing the temperature program rate from $1.5^{\circ}$ to $1.6^{\circ} / \mathrm{min}$ caused the elution temperature of naphthalene to rise by $5.5^{\circ}$. The corresponding increase in polarity proved to be slightly exaggerated, shifting the naphthalene peak into a group of poorly resolved minor components (chromatogram B, arrow). As a mild correction, the carrier gas pressure was raised by 0.05 bar, whereas the temperature program rate was not modified. This 

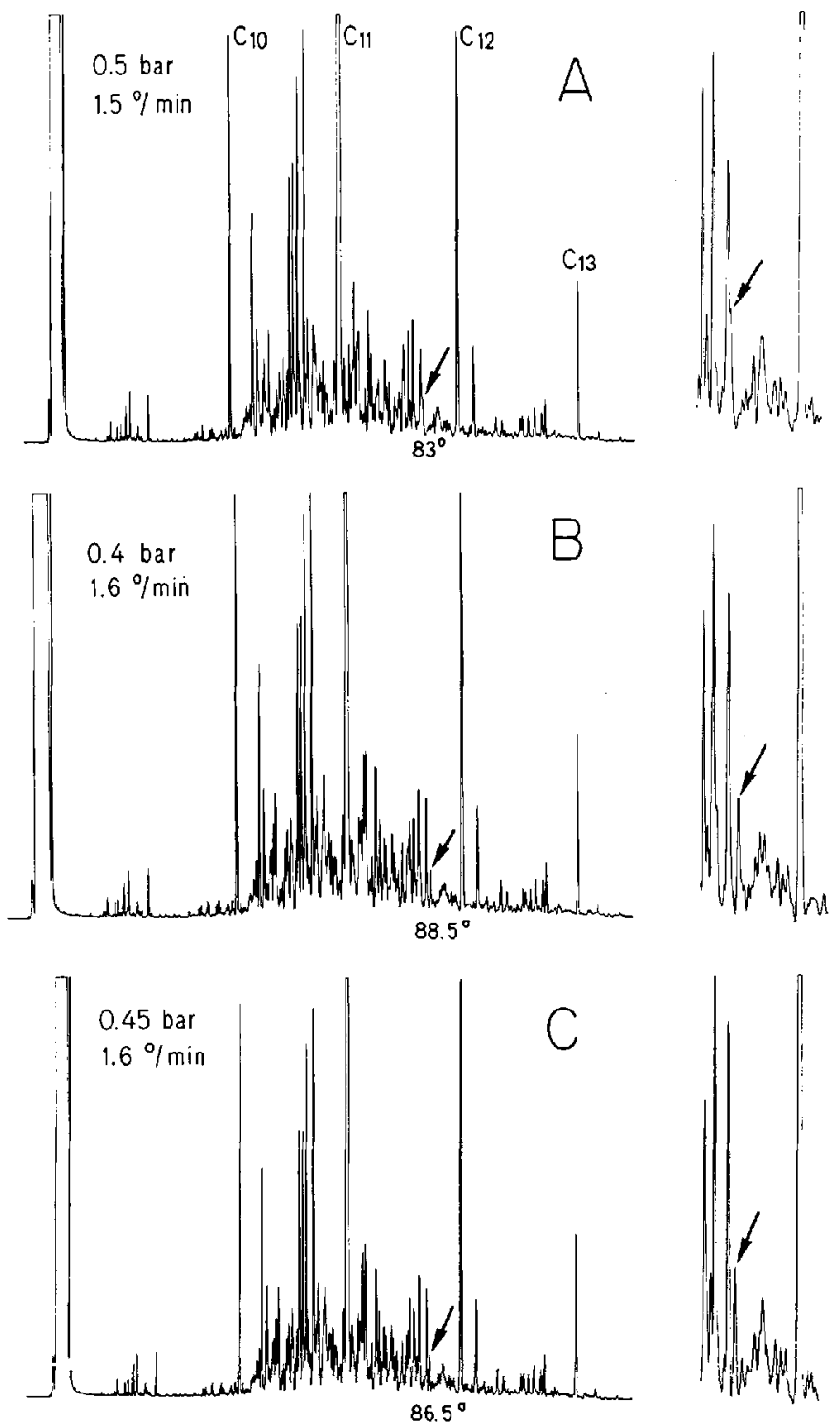

Fig. 4

Optimization of resolution of naphthalene in petrol fraction. Column $30 \mathrm{~m} \times 0.30 \mathrm{~mm}, 0.30 \mu \mathrm{m}$ PS-255. Sample dissolved in hexane. $1.5 \mu \mathrm{l}$ injected with splitting $1: 30$ at $50^{\circ}$. conditions see chromatograms. Arrow indicates naphthalene with corresponding elution temperature. Right hand side: section containing naphthalene, enlarged. For interpretation see text.

moved the naphthalene peak back into the ideal position without any coincidence (chromatogram C).

Figure 5 shows an example offering less fortunate conditions. 1,4-Dichlorobenzene (arrow) had to be determined in a waste water extract. At the bottom, the enlarged section of interest is shown, as obtained by 8 sets of carrier flow and temperature program rates, causing the elution temperature of dichlorobenzene to vary between $69^{\circ}$ and $86^{\circ}$. Visual comparison of the dichlorobenzene peaks hardly provides a clear preference for an elution temperature. Additional information is obtained by comparing the peak area of the dichlorobenzene peaks to the areas of two larger peaks left of dichlorobenzene ( $a$ and $b$ on the upper chromatogram). The corresponding area ratios are listed in Table II. Runs 1 and 7 show the smallest ratios, indicating minimum overlap with other substances.

\subsection{Optimizing the Information on Very Complex Samples}

Samples may be so complex (e.g. tobacco smoke) that even extreme resolution produces signals covering, on average, several substances. Accordingly, interpretation of mass spectra may be hard or impossible. Repeating the runs with slightly shifted elution temperatures, as described in the foregoing chapter, modifies the relative composition of the coeluted mixtures. The comparison of mass spectra obtained with different elution temperature permits assignment of the molecular and fragment ions to pure substances. Also co-chromatography with added substances becomes far more informative with this technique.

\subsection{Polarity and Film Thickness}

A major consequence of varying film thickness [16] is varying retention. This means varied column temperature under optimum isothermal conditions, and varied elution temperature with temperature programming. Both effects result in a varying column polarity. Thus, there is no exaggeration in the statement that a column with a different film thickness of the same stationary phase is basically a different column.

As a consequence of this fact, there has been sonsiderable discussion connected to our column testing procedure [17]. For a number of common stationary phases, we presented typical elution patterns of the test mixture. Repeatedly, important deviation from these patterns caused doubts and confusion. However, the disagreement was simply due to a variation in film thickness. Our standard fingerprints should no longer be used for peak assignment when the film thickness of the tested column differs substantially from the standard thickness of $0.15 \mu \mathrm{m}$.

Evidently, the range of variable polarity based on varying column (elution) temperature is limited, since excessive variation causes intolerable deviation from optimum chromatographic conditions. The range can be strongly extended by including film thickness as a variable parameter. As soon as, for instance, a working temperature producing a suitable polarity becomes excessively high for a given column, a column with a thicker film should be selected which behaves ideally under the given working temperature. Thus, film thickness is a very practical and efficient parameter for the optimization of the polarity of a given stationary phase.

Table II. Optimized resolution of 1,4-dichlorobenzene in a waste water extract; summary of parameters and results (see Figure 5).

\begin{tabular}{|c|c|c|c|c|c|}
\hline $\begin{array}{l}\text { rum } \\
\text { No. }\end{array}$ & $\begin{array}{c}\text { pressure } \\
\text { bar }\end{array}$ & $\begin{array}{l}\text { temp prog } \\
{ }^{\circ} \mathrm{C} / \mathrm{min}\end{array}$ & $\begin{array}{l}\text { elution } \\
\text { temp. }\end{array}$ & $\begin{array}{c}\text { peak are } \\
\text { peak a }\end{array}$ & $\begin{array}{l}\text { lative to } \\
\text { peak b }\end{array}$ \\
\hline 1 & 0.7 & 1.4 & 86 & 0.489 & 0.342 \\
\hline 2 & 0.8 & 1.3 & 82 & 0.520 & 0.358 \\
\hline 3 & 0.9 & 1.3 & 80 & 0.545 & 0.380 \\
\hline 4 & 0.9 & 1.2 & 78 & 0.610 & 0.418 \\
\hline 5 & 1.0 & 1.2 & 76 & 0.633 & 0.442 \\
\hline 6 & 1.0 & 1.1 & 75 & 0.518 & 0.362 \\
\hline 7 & 1.0 & 1.0 & 73 & 0.472 & 0.335 \\
\hline 8 & 1.2 & 1.0 & 69 & 0.625 & 0.432 \\
\hline
\end{tabular}



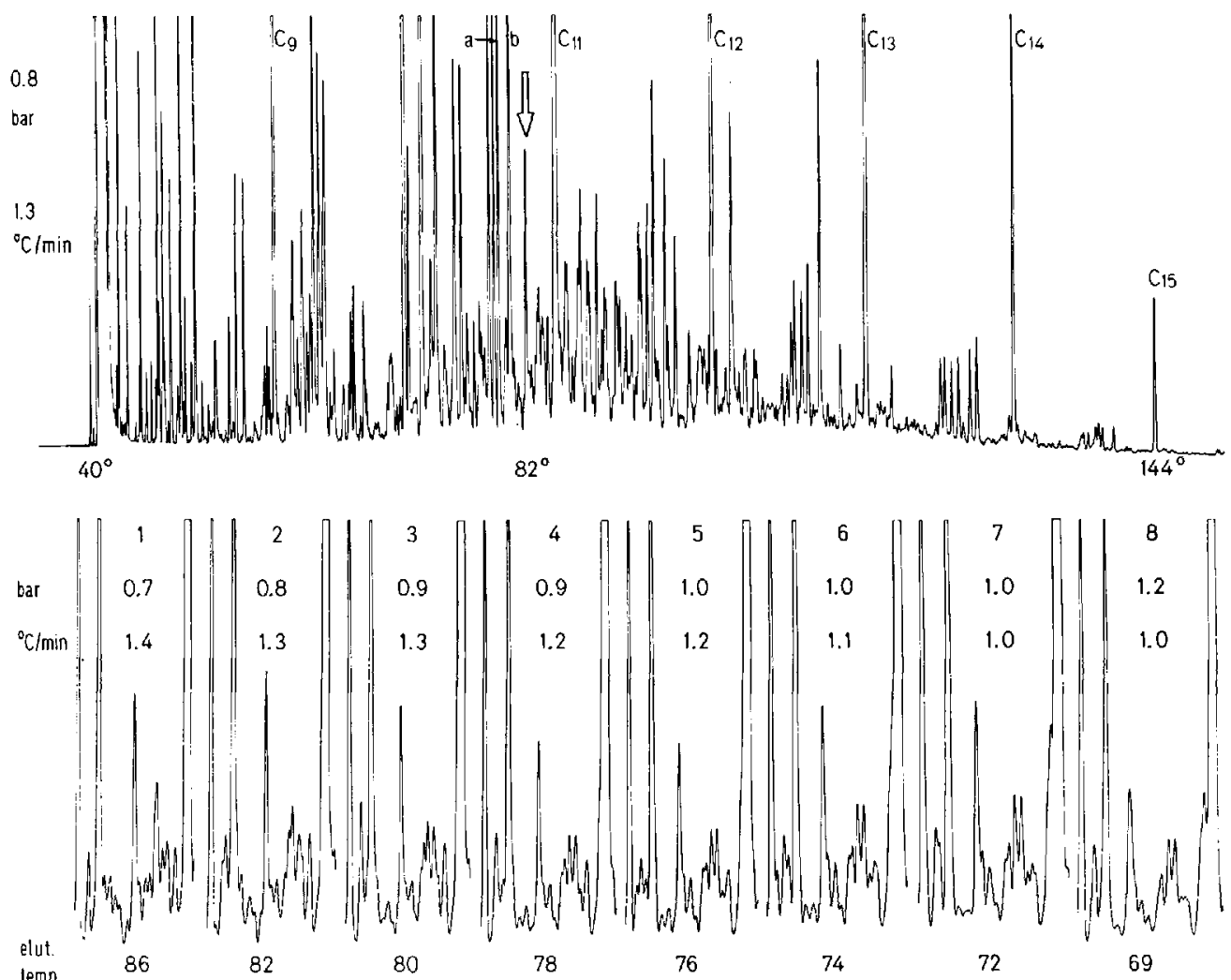

Fig. 5

Optimization of resolution of 1,4dichlorobenzene in waste water extract. Column $40 \mathrm{~m} \times 0.29 \mathrm{~mm}$, $0.40 \mu \mathrm{m} \mathrm{SE}-54$. Dichlorabenzene indicated with arrow, and elution temperature. Bottom: enlarged, dichloro benzene containing sections of 8 runs with systematically varied elution temperature. Run No. 2 corresponds to upper chromatogram.

\section{Acknowledgement}

Our work has again been sponsored by F. J. Burrus \& Cie, Boncourt, Switzerland.

\section{References}

[1] E. sz. Kovats, Advances in Chromatography, J. C. Giddings and R. A. Keller Editors, Marcel Dekker, Inc., New York 1965, Vol. 1, p. 234-235.

[2] L. Rohrschneider, ibidem, Vol. 4, New York 1967, p. 346, 348.

[3] G. Schomburg, ibidem, Vol. 6., New York 1968, p. 213.

[4] S. R. Lowry, H. B. Woodruff, T. L. Isenhour, J. Chromatogr. Sci. 14, $129(1976)$

[5] W. L. Zielinsky, D. E. Martire, Anal. Chem. 48, 1111 (1976).

[6] S. Rang, $K$. Kuningas, A. Orav, O. Eisen, J, Chromatogr. 128, 53 (1976).

17] D. Gross, H. Z. Weigel, Z. anal. Chem. 277, 31 (1975).
[8] L. Sojak, J. A. Rijks, J. Chromatogr. 119, 505 (1976).

[9] G. Schomburg, Gaschromatographie, Verlag Chemie, Taschentext Nr. 48, Weinheim 1977, p. 64,65.

[10] M. L. Lee, D. L. Vassilaros, C. M. White, M. Novotny, Anal, Chem. 51, 768 (1979).

[11] W. Jennings, Gas Chromatography with Glass Capillary Columns, II Edit., Academic Press, New York 1980, p. 121 , 122.

[12] E. Schulte, Praxis der Kapillar-Gaschromatographie, Springer, Heidelberg 1983 , p. 42, 82.

[13] H. Knöppel, M. de Bortoli, A. Peil, H. Vissers, V. Internat. Symposium on Cap. Chromatogr., Proceedings, Elsevier, Amsterdam 1983, p. 863.

[14] I. O. O. Korhonen, Chromatographia 17,195 (1983)

[15] I. O. O. Korhonen, Chromatographia 17, 70 (1983).

[16] K. Grob, G. Grob, HRC + CC 6, 133 (1983).

[17] K. Grob, G. Grob, K. Grob Jr., J. Chromatogr. 219, 13 (1981).

Received: July 6,1983

Accepted: July 19,1983 